\title{
Imagem corporal de adolescentes do sexo feminino e sua associação à composição corporal e ao comportamento sedentário
}

\author{
Body image of female adolescents and its association \\ with body composition and sedentary behavior
}

Núbia de Souza de Morais ${ }^{1}$

Valter Paulo Neves Miranda ${ }^{1}$

Silvia Eloiza Priore ${ }^{1}$

${ }^{1}$ Departamento de Nutrição e Saúde, Universidade Federal de Viçosa. Ed. Centro de Ciências Biológicas II, Campus Universitário. 36570.900 Viçosa MG Brasil. nubia.s.morais25@ gmail.com
Abstract The scope of this article is to evaluate the body image and its association with body composition, anthropometric indicators and sedentary behavior of female adolescents. It involved a cross-sectional study with female adolescents from Viçosa in the state of Minas Gerais. The body image was assessed by the Body Shape Questionnaire (BSQ) and by the Silhouette Scale. The body fat percentage (BFP) and other anthropometric parameters were measured. The sedentary behavior (SB) was evaluated by the sum of screen time (ST) and cellular time (CT). The sample consisted of 274 adolescents, the majority having an adequate BMI (81.6\%), however, 53.9\% had elevated BFP. More than half of the adolescents had elevated SB, $68.2 \%$ with ST and $54.7 \%$ with CT above $2 h$. Body dissatisfaction was expressed as 45.7\% by BSQ and $50.2 \%$ wished for a silhouette different than the current silhouette. The waist circumference $(W C)(O R=11.94, C I=2.744$ $51.728)$ and waist-to-height ratio (WHR) (OR = $10.535, C I=3.109$ - 35.698) were the measurements most associated with body dissatisfaction. All body composition measurements were significantly associated with body dissatisfaction, and the WC and WHR the most conditioning factors with dissatisfaction. The SB was elevated; however, it was not associated with a negative assessment of body image.

Key words Body image Adolescent, Body composition
Resumo $O$ objetivo deste artigo é avaliar a imagem corporal e sua associação à composição corporal, indicadores antropométricos e comportamento sedentário de adolescentes do sexo feminino. Estudo transversal com adolescentes do sexo feminino de Viçosa, MG. A imagem corporal foi avaliada por meio do Body Shape Questionnaire (BSQ) e pela Escala de Silhuetas. O percentual de gordura (\%GC) e outros parâmetros antropométricos foram aferidos. O comportamento sedentário (CS) foi avaliado a partir da soma do tempo de tela (TT) e do tempo de celular (TC). A amostra foi composta por 274 adolescentes, a maioria com IMC adequado (81,6\%), porém, 53,9\% apresentaram o \% GC elevado. Mais da metade das adolescentes apresentaram o CS elevado, 68,2\% com o TT e 54,7\% com o TC acima de $2 \mathrm{~h}$. A insatisfação corporal manifestou-se em 45,7\%, e 50,2\% desejaram uma silhueta diferente da atual. O perimetro da cintura $(P C)(O R=11,94, I C=2,744$ - 51,728) e a relação cintura-estatura ( $R C E)$ (OR $=10,535, I C=3,109-35,698)$ foram as medidas mais associadas à insatisfação corporal. Todas as medidas de composição corporal apresentaram associação significativa com a insatisfação corporal, sendo o PC e o RCE os fatores mais condicionantes. O CS mostrou-se elevado, no entanto, não se associou com a avaliação negativa da imagem corporal.

Palavras-chave Imagem corporal, Adolescente, Composição corporal 


\section{Introdução}

A Organização Mundial da Saúde (OMS) define a adolescência como um período de transição entre a infância e a idade adulta, englobando a faixa etária de10 a 19 anos $^{1}$. Durante esse período ocorrem alterações na composição corporal que podem influenciar todo o processo de formação da imagem corporal do indivíduo ${ }^{2}$. A sociedade, a mídia, a família e os amigos exercem forte pressão sobre a escolha de um padrão de beleza, na maioria das vezes com excessiva valorização do corpo magro, principalmente entre adolescentes do sexo feminino ${ }^{3,4}$.

A imagem corporal se refere à forma de avaliação que o indivíduo tem do tamanho e da forma corporal, bem como os sentimentos manifestados com a aparência física ${ }^{5}$. A insatisfação corporal é um dos componentes da dimensão atitudinal da Imagem Corporal, sendo frequentemente avaliado na população adolescente. As pesquisas têm demonstrado que a insatisfação corporal é frequente entre as meninas com excesso de peso e até mesmo naquelas que, mesmo no estado nutricional adequado, se percebem acima do peso $^{2,6,7}$.

As alterações que ocorrem na composição corporal durante a adolescência incluem variações nas proporções relativas de água, massa magra, massa mineral óssea, massa muscular esquelética e massa de gordura ${ }^{8,9}$. Fisiologicamente, o aumento do depósito de gordura corporal ocorre para que os hormônios de crescimento sejam liberados no organismo e, assim, estabelecer as condições para o estirão puberal ${ }^{10}$. A quantidade relativa de gordura é bem evidente nas meninas, pois esta aumenta progressivamente no decorrer da adolescência na região central ${ }^{11}$.

Outro aspecto relacionado com a composição corporal é o fato de muitos adolescentes estarem adotando um estilo de vida mais sedentário e/ou com maior ingestão de alimentos calóricos ${ }^{12}$. O comportamento sedentário (CS) pode ser avaliado pelo tempo assentado gasto em frente a um aparelho com tela (TV, vídeo game, computador, tablets e celular $)^{13}$. Estudos mostram que este comportamento pode estar substituindo o tempo destinado à prática de atividades físicas, que demandam maior gasto de energia, por atividades com baixo gasto energético, e assim, contribuindo para o aumento do sobrepeso e da obesidade $^{14,15}$. Atualmente, observa-se que crianças e adolescentes gastam entre $40 \%$ e $60 \%$ de suas horas do dia (fora do horário de sono) em atividades sedentárias ${ }^{13}$.
O CS está diretamente associado ao excesso de gordura corporal e, consequentemente, na manifestação de sentimentos negativos com a imagem corporal, principalmente entre as adolescentes, que adotam um estilo de vida menos ativo que os meninos, em função de mudanças no estilo de vida devido ao aumento das horas de estudo e do tempo realizando atividades mais sedentárias ${ }^{16-18}$. Não há na literatura um consenso em relação aos efeitos da atividade física na imagem corporal ${ }^{19}$.

Percebe-se a necessidade de se investigar diferentes aspectos da imagem corporal associados com as medidas de composição corporal e comportamentos relacionados com o sobrepeso e obesidade. Sendo assim, o estudo se propôs avaliar a imagem corporal e sua associação ao comportamento sedentário, à composição corporal e a outros indicadores antropométricos de adolescentes do sexo feminino.

\section{Métodos}

Trata-se de um estudo transversal descritivo.

\section{População e amostra}

A população do estudo foi composta por 274 adolescentes, de 14 a 19 anos, do sexo feminino, residentes no município de Viçosa-MG e regularmente matriculadas em uma escola da rede pública do município.

A amostra deste estudo foi selecionada por conveniência, pelo fato de pertencer a um maior intitulado "Avaliação do estilo de vida, dos fatores de risco cardiometabólicos, da microbiota intestinal e da imagem corporal de adolescentes do sexo feminino", aprovado pelo Comitê de Ética em Pesquisa com Seres Humanos da UFV.

No entanto, para garantir a significância das análises, foi realizado o cálculo do poder do estudo por meio do programa OpenEpi $i^{\circledR}$, versão 3, levando-se em consideração o número de adolescentes expostos e não expostos com a insatisfação corporal, avaliada pelo questionário de autoavaliação Body Shape Questionnaire - (BSQ). Verificou-se que o poder foi de $87,49 \%$ com a correção de continuidade.

Para a participação na pesquisa, a adolescente deveria ter entre 14 e 19 anos, já ter apresentado menarca no mínimo há um ano, entregar assinado o Termo de Consentimento Livre e Esclarecido (TCLE) e o Termo de Assentimento (TA), estar presente nos procedimentos de coletas, não 
fazer uso de algum remédio controlado ou estar participando de outra pesquisa que envolvesse avaliação da composição corporal ou do controle do estado nutricional.

As coletas de dados foram realizadas primeiramente na escola e, em um segundo momento, na Divisão de Saúde (DSA) da Universidade Federal de Viçosa (UFV). A escola foi informada anteriormente sobre a realização do estudo e somente após o consentimento houve contato com as alunas para explicação detalhada dos procedimentos que foram realizados.

Neste contato, as alunas ficaram cientes do estudo e foi disponibilizado o TCLE, que deveria ser entregue e assinado, por ela mesma e por seu responsável, e o TA, que deveria ser entregue e assinado pela adolescente, como condição à participação no estudo. A participação foi voluntária, podendo a participante abandonar a pesquisa a qualquer momento, sem nenhum prejuízo.

Após a entrega dos termos, foram coletadas informações sociodemográficas (identificação das adolescentes) e dados antropométricos como peso, gordura corporal (\%GC) e estatura. Essas medidas foram realizadas em um espaço cedido pela escola, que garantia a privacidade das avaliações. Nesse contato, também foi avaliada a imagem corporal das adolescentes por meio da escala de silhuetas ${ }^{20}$. Na etapa seguinte, as alunas compareceram a DSA da UFV, onde a avaliação antropométrica foi realizada, todas estas medidas realizadas em triplicada, obtendo a média das duas medidas mais próximas. Nesta etapa ocorreu também a aplicação do BSQ.

\section{Instrumentos e procedimentos}

Os instrumentos selecionados para avaliação da imagem corporal foram a Escala de Silhuetas para Adultos Brasileiros ${ }^{21}$, validada para adoles$\operatorname{centes}^{20}$ (Figura 1) e o questionário de Imagem Corporal, o-BSQ ${ }^{22}$.

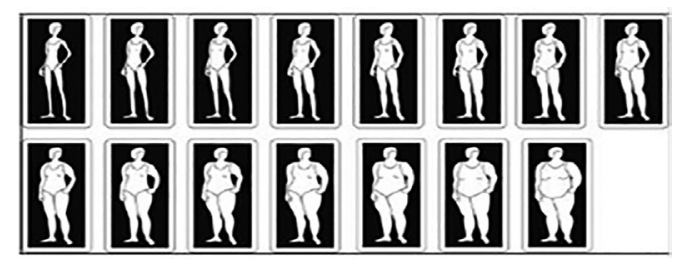

Figura 1. Escala de Silhuetas para Adultos Brasileiros de Kakeshita et al. ${ }^{21}$. Validada para adolescentes brasileiros por Laus et al. ${ }^{20}$.
A Escala de Silhuetas é composta por 15 figuras, em forma de cartões plastificados, enumeradas no verso. As figuras foram criadas considerando a altura média brasileira de $1,72 \mathrm{~m}$ para homens e 1,65 m para mulheres. Os valores médios de IMC correspondentes a cada figura variaram de 12,5 a $47,5 \mathrm{~kg} / \mathrm{m}^{2}$, com diferença constante de 2,5 pontos.

A escala foi apresentada de forma ordenada ascendente e a voluntária deveria primeiro escolher a figura que melhor representava seu corpo atualmente, depois, escolher a figura que melhor representava o corpo que ela gostaria de ter, podendo ser escolhidas as mesmas figuras ou não. No caso de serem selecionadas as mesmas figuras, o indivíduo foi classificado como satisfeito. Quando a figura escolhida como o corpo desejado apresentou numeração maior do que a escolhida como a atual, considerou-se que a participante teve o desejo de aumentar o tamanho do seu corpo, e quando essa figura teve numeração menor, considerou-se o desejo em diminuir o tamanho corporal ${ }^{20}$.

O BSQ é um questionário que avalia a insatisfação com o peso e a forma corporal nas últimas 4 semanas. Possui 34 questões com respostas na forma de escala Likert de pontos, que são respondidos pelo próprio participante. Esse instrumento tem a finalidade de verificar a preocupação com o peso e a forma corporal. Cada pergunta do questionário possui opções de repostas de 1 a 6, sendo: 1 - nunca; 6 - sempre e 4 níveis de insatisfação com a aparência física de acordo com a pontuação final $(<80$ pontos - não apresenta insatisfação corporal; de 80 a 110 pontos - apresenta leve insatisfação; de 110 a 140 pontos - moderada insatisfação; $\geq 80$ pontos - indica grave insatisfação) $)^{22}$.

Para avaliar a composição corporal, foram realizadas medidas do peso, estatura, percentual de gordura corporal, perímetro da cintura e dobras cutâneas. Além dos cálculos do IMC e relação cintura-estatura.

O peso foi aferido em balança digital eletrônica da marca Tanita BC-543 ${ }^{\circledR}\left(\mathrm{TMAB}^{\circledR}{ }^{\circledR}\right.$, Londres, Reino Unido), e para a estatura foi utilizado o estadiômetro portátil (Alturexata ${ }^{\circledR}$, Belo Horizonte, Brasil). Foi calculado o Índice de Massa Corporal (IMC) por sendo classificado por meio do software Who AnthroPlus ${ }^{23}$.

O percentual de gordura corporal foi aferido utilizando-se a balança Tanita BC-543 ${ }^{\circledR}\left(\mathrm{TMAB}^{\circledR}\right.$, Londres, Reino Unido), classificado considerando pontos de corte específicos para adolescentes do sexo feminino: $<20 \%$ baixo peso; $\geq 20$ e $<$ 
$25 \%$ eutrofia; $\geq 25 \%$ e $<30 \%$ risco de sobrepeso; $\geq 30 \%$ sobrepeso $^{24}$.

O perímetro da cintura (PC) foi medido utilizando-se uma fita métrica inelástica (Cardio$\operatorname{med}^{\circledR}$, São Luis, MA, Brasil) e flexível. Foi aferido no ponto médio entre a margem inferior da última costela e a crista ilíaca, no plano horizontal ${ }^{25}$, utilizando-se como ponto de corte o percentil 90 (P90) da própria população ${ }^{26}$. A RCE foi obtida pela relação entre a medida da cintura $(\mathrm{cm})$ e a medida da estatura $(\mathrm{cm})$, tendo como ponto de corte $\mathrm{PC}<0,5$.

As dobras cutâneas tricipital, bicipital, suprailíaca e subescapular foram aferidas através das técnicas propostas pela $\mathrm{WHO}^{23}$. A dobra cutânea tricipital foi medida na parte posterior do antebraço, sobre o músculo tríceps, no ponto médio entre o acrômio e o olécrano. A dobra cutânea bicipital foi aferida na parte anterior do antebraço, sobre o músculo bíceps, tomando como referência o mesmo ponto considerado para a aferição da dobra cutânea tricipital. A dobra cutânea suprailíaca foi acima da crista ilíaca, seguindo a linha axilar média. E a dobra cutânea subescapular foi aferida logo abaixo da extremidade da escápula, sendo angulada em $45^{\circ}$ a partir do plano horizontal.

O CS foi avaliado por meio de um questionário de avaliação do Tempo de Tela (TT) de Barros e Navas ${ }^{27}$. A adolescente anotou o tempo gasto em um dia da semana assistindo TV, jogando videogame, usando o computador e tablet. O tempo de tela do celular (TC) foi acrescentado após as adolescentes relatarem uso contínuo desse aparelho. O CS foi avaliado a partir da soma do tempo do uso dos aparelhos, sendo analisado separadamente o TT e o TC para não ocorrer sobreposição dos tempos. Essa medida foi tomada após as próprias participantes informarem que muitas vezes usavam o celular usando outro aparelho de tela, como por exemplo, assistindo TV. Para categorização do CS foi considerado TT e TC elevados a soma maior que duas horas ${ }^{28}$.

\section{Análise estatística}

O banco de dados foi elaborado no software Excel e teve dupla entrada/digitação. As análises estatísticas foram realizadas com o auxílio do software Statistical Package for the Social Sciences (SPSS, IBM ${ }^{\circledR}$ Canadá) for Windows, versão 17.0. Foram aplicados testes paramétricos ou não paramétricos, de acordo com a distribuição das variáveis, bem como testes de associação, correlação e análise de regressão. O nível de rejeição da hipótese de nulidade foi de $\alpha=5 \%$.
O teste de normalidade de Kolmogorov-Smirnov foi utilizado para verificar a distribuição dos valores das variáveis quanto aos afastamentos observados em relação aos esperados na distribuição normal. A partir deste teste verificou-se que as variáveis não apresentaram distribuição normal, com isso foram utilizados testes não paramétricos.

Para a análise descritiva das variáveis foram apresentados valores de média, desvio-padrão e amplitude, mediana, intervalo interquartílico das variáveis.

Os testes do qui-quadrado de Pearson e de Fisher foram utilizados para verificar a associação entre duas variáveis qualitativas ${ }^{29}$. A correlação de Spearman foi usada para verificar a correlação entre duas variáveis numéricas quantitativas ${ }^{29}$.

O teste de Kruskal-wallis foi utilizado para a avaliação da variância de pontuação do BSQ entre três grupos ou mais. Para a complementação deste teste foram realizados testes comparativos entre duas variáveis para verificar a diferença significativa, baseando-se na correção de Bonferroni - $/ \mathrm{K}$ ( = 0,05, K = número de comparações). O teste de Mann Whitney foi utilizado para a análise entre duas amostras independentes ${ }^{30}$.

O teste de regressão logística binária foi aplicado para a análise da associação entre a insatisfação corporal avaliada pela escala de silhuetas e as variáveis de composição corporal, sendo ambas categorizadas em dois grupos para atenderem as exigências do teste. Como complemento de análise foram interpretados os valores da razão de chance Odds Ratio (OR), e intervalo de confiança de $95 \%$, para verificar o quanto uma variável da composição corporal pode influenciar na insatisfação corporal avaliada pela escala de silhuetas.

\section{Resultados}

Participaram da pesquisa 274 adolescentes do sexo feminino. A Tabela 1 apresenta uma análise descritiva das variáveis: idade, medidas antropométricas e de composição corporal.

Segundo o IMC, $81,6 \%$ das adolescentes encontrava-se eutrófica. No entanto, segundo a avaliação do \%GC, os percentuais de adolescentes eutróficas, com risco de sobrepeso e sobrepeso foram próximos, $29,6 \%, 29,2 \%$ e $24,7 \%$, respectivamente. Ou seja, 53,9\% apresentaram \%GC elevado. Somando o \%GC das dobras cutâneas periféricas e dobras cutâneas centrais verificouse uma média de $32,18 \%$, valor considerado como excesso de adiposidade corporal. A maioria 
apresentou PC e RCE, 84,6\% e 80,5\%, respectivamente (Tabela 2).

Em relação ao CS, a maioria das alunas apresentou TT $(68,2 \%)$ e TC $(54,7 \%)$ elevados. Não foi observada relação entre o TT e o TC com a composição corporal das adolescentes.

Pela pontuação do BSQ verificou-se que $45,7 \%$ apresentaram algum grau de insatisfação, dessas 3,4\% com grave insatisfação. Grande parte das adolescentes (40,5\%) deu a resposta "sempre” para a questão 34 do BSQ, a qual avalia se a preocupação com seu físico faz sentir-se que deveria fazer exercícios.

Com relação à escala de silhuetas, 50,2\% das meninas se mostraram insatisfeitas com a imagem corporal, sendo que 35,6\% apresentaram insatisfação negativa, ou seja, elas tiveram desejo em obter uma silhueta menor que a atual, já $14,6 \%$ insatisfação positiva, que diz respeito ao desejo de obter uma silhueta maior que a atual.

A figura mais escolhida da escala de silhuetas para representar a silhueta atual foi a $03(20,2 \%)$ seguida pela figura 04 (17,2\%). Da mesma forma, a silhueta mais selecionada como ideal foi a 03 $(23,6 \%)$, seguida pela silhueta $04(23,2 \%)$.

A avaliação da relação entre as medidas de composição corporal e insatisfação mostrou que quanto maior os valores de IMC, \%GC, PC e RCE maior foi a pontuação do BSQ (Figura 2).
Tabela 2. Classificação do estado nutricional segundo IMC, gordura corporal, perímetro da cintura e relação cintura - estatura. Viçosa, MG - 2014/2015.

\begin{tabular}{lrr}
\hline Variáveis de composição corporal & n & $\%$ \\
\hline IMC & 8 & 3,0 \\
$\quad$ Baixo peso & 218 & 81,6 \\
Eutrófico & 40 & 15,0 \\
Sobrepeso/ Obesidade & & \\
\%GC & 42 & 15,7 \\
$\quad$ Baixo peso & 79 & 29,6 \\
$\quad$ Eutrófico & 78 & 29,2 \\
$\quad$ Risco de sobrepeso & 66 & 24,7 \\
$\quad$ Sobrepeso & & \\
PC & 226 & 84,6 \\
$\quad$ Adequado & 25 & 9,4 \\
$\quad$ Elevado & & \\
RCE & 215 & 80,5 \\
$\quad$ Adequada & 31 & 11,6 \\
$\quad$ Elevada &
\end{tabular}

IMC: Índice de massa corporal; \%GC: percentual de gordura corporal; PC: Perímetro da cintura; RCE: Relação cintura-estatura; \%: Frequência relativa; n: Frequência absoluta.

Houve diferença significativa dos valores de insatisfação corporal entre os grupos de classificação do IMC - eutrófico e sobrepeso $(\mathrm{p}<0,01)$,

Tabela 1. Análise descritiva das variáveis idade, medidas antropométricas e de composição corporal de adolescentes do sexo feminino de Viçosa, MG - 2014/2015.

\begin{tabular}{lrrrrrr}
\hline \multicolumn{1}{c}{ Variáveis descritivas } & Média & Mediana & DP \pm & Amplitude & Mínimo & Máximo \\
\hline Idade (anos) & 15,60 & 16,00 & 1,26 & 6,00 & 14,00 & 19,00 \\
Peso (kg) & 57,09 & 54,45 & 12,45 & 85,40 & 36,10 & 121,50 \\
Estatura (cm) & 161,95 & 161,65 & 6,15 & 41,60 & 146,00 & 187,60 \\
IMC (kg/m²) & 21,73 & 20,85 & 4,39 & 32,12 & 15,10 & 47,22 \\
GC (\%) & 26,32 & 25,60 & 6,73 & 47,10 & 5,00 & 52,10 \\
PC (cm) & 72,27 & 70,50 & 9,80 & 90,50 & 30,50 & 121,00 \\
PQ (cm) & 93,26 & 92,75 & 9,27 & 108,75 & 29,75 & 138,50 \\
DC suprailíaca (mm) & 20,24 & 18,50 & 8,79 & 43,00 & 7,00 & 50,00 \\
DC subescapular (mm) & 14,99 & 13,50 & 6,40 & 38,00 & 7,00 & 45,00 \\
DC bicipital (mm) & 11,41 & 10,00 & 5,78 & 42,50 & 3,50 & 46,00 \\
DC tricipital (mm) & 20,67 & 20,00 & 6,75 & 41,00 & 9,00 & 50,00 \\
DC periférica (\%) & 11,72 & 10,34 & 5,76 & 42,48 & 3,79 & 46,27 \\
DC central (\%) & 20,46 & 18,72 & 8,79 & 43,02 & 7,21 & 50,23 \\
RCE & 0,45 & 0,43 & 0,06 & 0,56 & 0,19 & 0,75 \\
RCQ & 0,78 & 0,77 & 0,11 & 1,83 & 0,34 & 2,17 \\
\hline
\end{tabular}

IMC: Índice de massa corporal; \%GC: percentual de gordura corporal; PC: Perímetro da cintura; PQ: Perímetro do quadril; DC: Dobra cutânea; RCE: Relação cintura-estatura; RCQ: Relação cintura-quadril. 


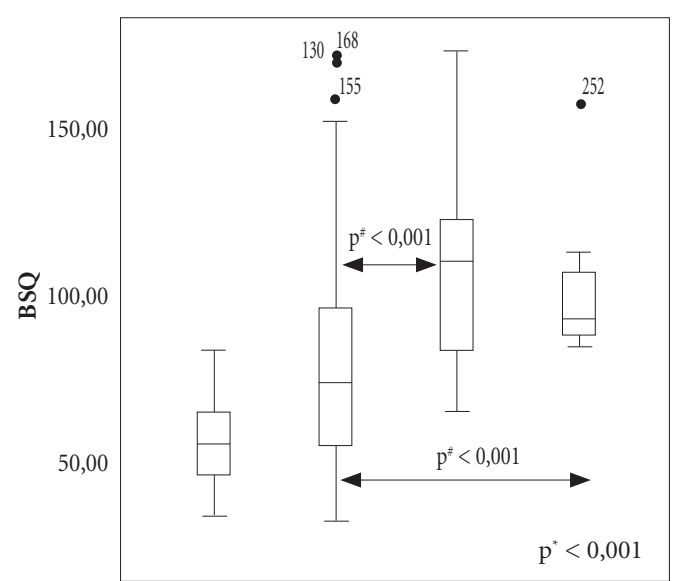

Baixo peso Eutrófico Sobrepeso Obesidade

Classificação IMC

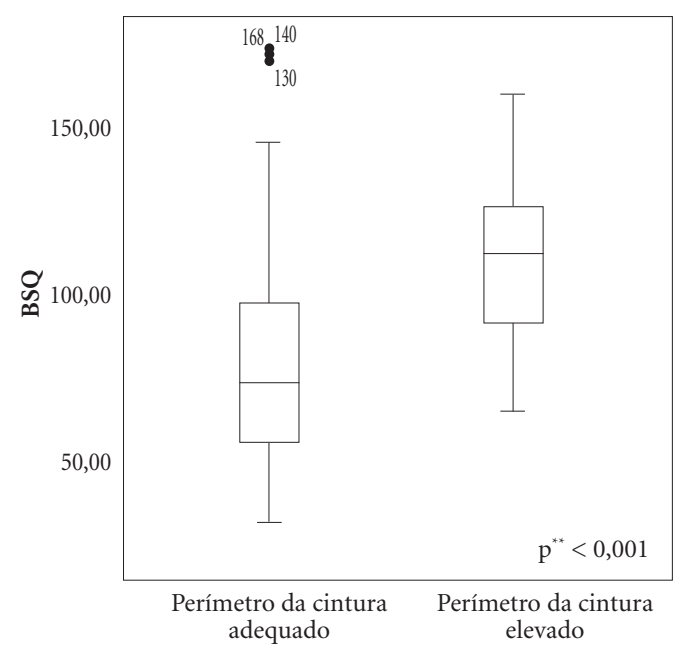

Classificação perímetro da cintura

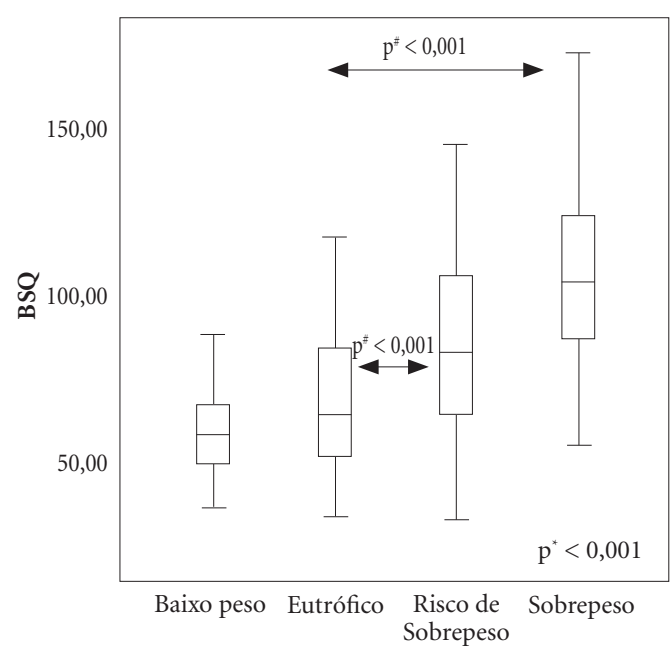

Classificação percentual de gordura

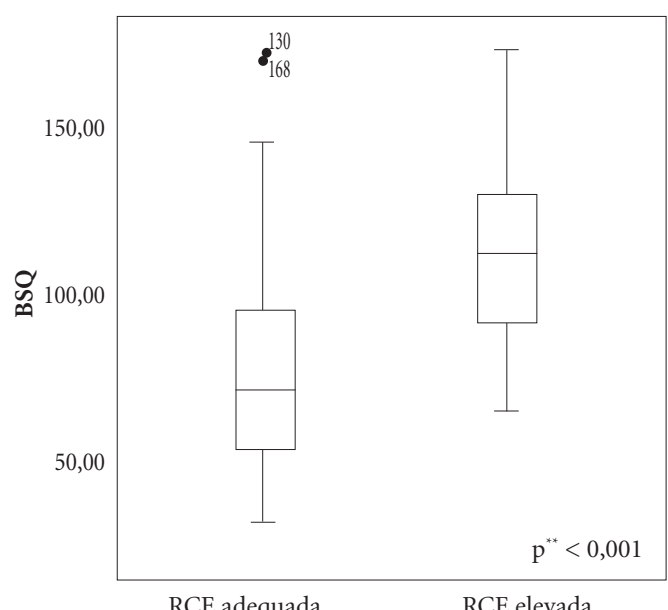

Classificação RCE

Figura 2. Gráficos Box Plot das variáveis de composição corporal em relação ao BSQ.

BSQ: Body Shape Questionnaire; RCE: Relação cintura-estatura. ${ }^{\star}$ Teste de Kruskal-Wallis. ${ }^{\star *}$ Teste de Mann-Whitney. ${ }^{*} \mathrm{p}<0,016$-Correção de Bonferroni.

eutrófico e obesidade (p < 0,01); \%GC - \%GC adequado e risco de sobrepeso ( $p<0,01), \%$ GC adequado e sobrepeso ( $\mathrm{p}<0,01)$; PC - PC adequado e PC elevado ( $p<0,01)$; RCE - RCD adequado e RCE elevado $(\mathrm{p}<0,01)$ (Tabela 3 ).

A associação entre composição corporal e a insatisfação corporal segundo a escala de silhuetas, avaliada pela regressão logística binária, mostrou que adolescentes com sobrepeso/obesidade, PC e RCE elevados apresentaram maiores chances de serem insatisfeitas com a imagem corporal. Sendo que as medidas que mais se associaram com a insatisfação foram o PC $(\mathrm{OR}=11,94, \mathrm{IC}=$ $2,744-51,728)$ seguido pelo RCE $(\mathrm{OR}=10,535$, IC $=3,109-35,698)($ Tabela 4).

\section{Discussão}

O estudo mostrou que todas as medidas de avaliação da composição corporal apresentaram associação com a manifestação da insatisfação corporal, tanto pela avaliação do BSQ quanto pela avaliação da Escala de Silhueta. Mais da metade 
Tabela 3. Avaliação da relação entre as medidas de composição corporal e insatisfação corporal avaliadas por meio do BSQ. Viçosa, MG - 2014/2015.

\begin{tabular}{|c|c|c|c|}
\hline Medidas de composição corporal & Mediana & Intervalo interquartílico & \\
\hline IMC & & & $\mathbf{p}^{\star}$ \\
\hline Baixo peso & 56,00 & $47-74$ & $<0,001$ \\
\hline Eutrófico & 75,00 & $56-97,25$ & \\
\hline Sobrepeso & 111,00 & $84-124$ & \\
\hline Obesidade & 93,00 & $89-113$ & \\
\hline$\% \mathrm{GC}$ & & & $\mathbf{p}^{*}$ \\
\hline Baixo peso & 59,00 & $51-69$ & $<0,001$ \\
\hline Eutrófico & 65,50 & $52,75-85$ & \\
\hline Risco de sobrepeso & 84,00 & $64,5-106,25$ & \\
\hline Sobrepeso & 105,00 & $87,5-124,5$ & \\
\hline $\mathrm{PC}$ & & & $\mathrm{p}^{* *}$ \\
\hline Adequado & 75,00 & $56-99$ & $<0,001$ \\
\hline Elevado & 113,00 & $91,5-131$ & \\
\hline $\mathrm{RCE}$ & & & $\mathrm{p}^{* *}$ \\
\hline Adequada & 73,50 & $56-96$ & $<0,001$ \\
\hline Elevada & 113,00 & $113-133$ & \\
\hline
\end{tabular}

IMC: Índice de massa corporal; \%GC: percentual de gordura corporal; PC: Perímetro da cintura; RCE: Relação cintura-estatura; BSQ: Body Shape Questionnaire. ${ }^{\star}$ Teste de Kruskal-Wallis. ${ }^{*}$ Teste de Mann-Whitney. ${ }^{*} \mathrm{p}<0,016$-Correção de Bonferroni.

Tabela 4. Relação entre composição corporal e imagem corporal avaliada pela escala de silhuetas. Viçosa, MG 2014/2015.

\begin{tabular}{|c|c|c|c|c|c|c|c|}
\hline \multicolumn{2}{|l|}{ Satisfeito (\%) } & \multirow{2}{*}{ Insatisfeito (\%) } & \multirow[t]{2}{*}{ Total } & \multirow[t]{2}{*}{ OR } & \multicolumn{2}{|c|}{ IC $95 \%$} & \multirow[t]{2}{*}{$\mathbf{p}$} \\
\hline IMC & & & & & & & \\
\hline Baixo Peso - Eutrófico & $119(44,7)$ & $107(40,2)$ & $226(85)$ & 1 & & & \\
\hline Sobrepeso - Obesidade & $6(2,3)$ & $34(12,8)$ & $40(15)$ & 6,302 & 2,546 & 15,599 & 0,000 \\
\hline Total & $125(47)$ & $141(53,0)$ & $266(100)$ & & & & \\
\hline \multicolumn{8}{|l|}{ Percentual de Gordura } \\
\hline Baixo Peso - Eutrófico & $78(29,4)$ & $44(16,6)$ & $122(46)$ & 1 & & & \\
\hline Risco de Sobrepeso - Sobrepeso & $46(17,4)$ & $97(36,6)$ & $143(54)$ & 3,738 & 2,246 & 6,222 & 0,000 \\
\hline Total & $124(46,8)$ & $141(53,3)$ & $265(100)$ & & & & \\
\hline \multicolumn{8}{|l|}{ Perímetro da Cintura } \\
\hline PC Adequado & $115(45,8)$ & $111(42,2)$ & $226(90)$ & 1 & & & \\
\hline PC Elevado & $2(0,8)$ & $23(9,2)$ & $25(10)$ & 11,94 & 2,744 & 51,728 & 0,000 \\
\hline Total & $117(46)$ & $134(53,4)$ & $251(100)$ & & & & \\
\hline \multicolumn{8}{|l|}{ RCE } \\
\hline RCE Adequado & $114(46,3)$ & $101(41,1)$ & $215(87,4)$ & 1 & & & \\
\hline RCE Elevado & $3(1,2)$ & $28(11,4)$ & $31(12,6)$ & 10,535 & 3,109 & 35,698 & 0,000 \\
\hline Total & $117(47,6)$ & $129(52,4)$ & $246(100)$ & & & & \\
\hline
\end{tabular}

das meninas avaliadas foram identificadas com comportamento sedentário elevado (TT e CT > 2h), no entanto, este não se mostrou associado com a imagem corporal.

O CS foi elevado na maioria das participantes, tanto pela avaliação do TT, quanto pelo TC. Entretanto, não foi observada relação entre o TT e TC com a composição corporal das meninas, resultado também observado por Santos et al. ${ }^{28}$. A avaliação do CS por meio do tempo de tela é importante, pois, segundo Almeida et al. ${ }^{31}$, maior tempo gasto em frente a aparelhos eletrônicos favorece o aumento do sedentarismo, além de contribuir para o desenvolvimento de hábitos alimentares inadequados, podendo levar ao sobrepeso e obesidade ${ }^{15,32}$. 
A maioria das adolescentes foi classificada como eutrófica pelo IMC, por outro lado, mais da metade apresentaram o \%GC elevado ${ }^{24}$. Resultados semelhantes aos encontrados por Faria et al. ${ }^{33}$, que ao analisarem o estado nutricional de adolescentes do sexo feminino, também verificaram que a maioria estava eutrófica de acordo com o IMC, porém, grande parte das adolescentes apresentaram elevado $\% \mathrm{GC}$, sendo que $48 \%$ delas estavam em risco de sobrepeso e $61 \% \mathrm{com}$ sobrepeso. Dessa forma, ressalta-se a importância em se avaliar o \%GC juntamente com o IMC para uma avaliação mais completa da composição corporal.

Assim como outros resultados encontrados na literatura, foi constatado que as adolescentes com sobrepeso, obesidade e maior adiposidade corporal foram mais propensas a apresentarem insatisfação com a própria imagem ${ }^{2,34}$. Miranda et al. ${ }^{35}$ também encontraram associação entre o IMC e \%GC com a insatisfação corporal em adolescentes do sexo feminino.

Tal fato pode ser explicado devido à forte influência que a mídia e a sociedade em geral exercem sobre a população, impondo um padrão de beleza que muitas vezes é inatingível. Além disso, o fato das mulheres serem mais insatisfeitas pode ser explicado por fatores psicológicos, biológicos e socioculturais. Importante observar que as questões socioculturais afetam as meninas, as quais muitas vezes são estimuladas a praticarem atividades físicas e a fazerem dietas para perderem peso. Tais influências podem contribuir para o desenvolvimento de distintos padrões estéticos e percepções em relação ao próprio corpo ${ }^{36}$.

A insatisfação corporal foi encontrada em $45,7 \%$ das alunas pela avaliação do BSQ e em $50,2 \%$ pela Escala de Silhuetas. Em relação ao $\mathrm{BSQ}$, observou-se que quanto maiores os valores do IMC, \%GC, PC e RCE maior a pontuação de insatisfação. Assim como em outros estudos que também verificaram, por meio de análise do BSQ, que adolescentes com sobrepeso e obesidade apresentavam maiores índices de insatisfação, comparando-se aos que se encontravam eutróficos e com baixo peso ${ }^{2,35}$.

Constatou-se que as medidas de PC (OR $=11,94, \mathrm{IC}=2,744-51,728)$ e a $\mathrm{RCE}(\mathrm{OR}=$ $10,535$, IC $=3,109-35,698)$ foram as que mais se associaram à insatisfação corporal. Pedroni et al. ${ }^{37}$ também observaram em seu estudo que entre os escolares avaliados com insatisfação corporal, a maioria apresentou perímetro da cintura elevado. Considerando que essas medidas representam a gordura central, ou seja, localizada na região abdominal, a ocorrência de insatisfação associada a essas medidas pode ser devido à estética, já que atualmente a gordura é associada à falta de beleza, e as mulheres cada vez mais buscam um corpo magro, a fim de atingirem um padrão de beleza imposto pela sociedade e se sentirem bonitas.

Em relação à insatisfação por partes corporais, Amaral et al..$^{38}$ verificaram que a "área" peso obteve a menor média de satisfação corporal entre as meninas com 3,19 média de pontos. Glaner et al. ${ }^{39}$ verificaram que as meninas com adiposidade elevada, avaliada pelo somatório das dobras cutâneas bicipital e tricipital, apresentou 1,95 (IC95\% $=1,17-3,25)$ vezes mais chance de insatisfação com a imagem corporal do que aquelas com adiposidade adequada. Percebe-se que estes estudos que avaliam a associação entre imagem corporal e diferentes indicadores antropométricos publicados não foram realizados com instrumentos devidamente validados para a população adolescente brasileira.

Outro dado interessante encontrado nesse questionário foi que 111 meninas (40,5\%) marcaram a resposta sempre (6) para a questão de número 34 "A preocupação com seu físico faz-lhe sentir que deveria fazer exercícios?". Com isso, percebe-se como as meninas sentem a necessidade de praticar atividade física devido à preocupação com a aparência física, porém, na prática, gastam bastante tempo realizado atividades sedentárias, fato observado neste estudo na avaliação do TT e do TC.

A análise da escala de silhuetas mostrou que, entre as alunas que apresentaram insatisfação corporal, 35,6\% apresentou insatisfação negativa, ou seja, elas tiveram desejo em obter uma silhueta menor que a atual. Outra parcela $(14,6 \%)$ mostrou insatisfação positiva, que diz respeito ao desejo em aumentar a silhueta. Outros autores também encontraram em seu estudo que entre as adolescentes insatisfeitas com a imagem corporal, a maioria $(71,7 \%)$ queria reduzir o tamanho do corpo $^{2,40}$. Esses resultados eram esperados, pois como já citado na literatura, as mulheres tendem a buscar um corpo mais magro ${ }^{41,42}$.

A figura mais escolhida entre as adolescentes como a silhueta ideal foi a de número 03 , que representa um IMC de $17,5 \mathrm{~kg} / \mathrm{m}^{2}$, este valor de IMC pode ser considerado como baixo para as adolescentes, segundo a classificação da Organização Mundial da Saúde ${ }^{43}$.

O desenho transversal pode ser considerado uma limitação deste estudo, pois não permitiu uma avaliação de causa e efeito dos fatores que 
se associaram com a insatisfação corporal. No entanto, verificou-se que diferentes variáveis da composição corporal se associaram com a insatisfação, sendo as medidas de avaliação da gordura central, PC e RCE, aquelas que apresentaram maior associação.

Além disso, a utilização da balança, por ser um método duplamente indireto para a aferição do \%GC poderia ser considerado uma limitação, porém, Gonçalves et al. ${ }^{44}$, que compararam o uso da bioimpedância vertical (BIA) - marca Tanita em relação ao DEXA (Lunar Prodigy Advance DEXA System), verificaram que a BIA apresentou valores semelhantes para a composição corporal quando comparada ao DEXA. Elas observaram também que o uso de protocolo nas avaliações não influenciou nos resultados.

São escassos na literatura estudos que avaliem a relação entre o comportamento sedentário e a imagem corporal dos adolescentes, isso é importante pelo fato do primeiro contribuir para o sobrepeso e a adiposidade gordura corporal por deixarem as adolescentes menos fisicamente ativas $^{19}$. A avaliação de vários aparelhos que possuem tela (TV, videogame, computador, tablet e celular) é uma alternativa tomada para analisar de forma mais completa o comportamento sedentário adotado, visto que muitos estudos avaliam apenas o tempo em frente à televisão ${ }^{14,45}$.

A partir destas constatações, percebe-se a necessidade de se avaliar o CS e o nível de atividade física habitual, pelo menos três dias na semana, para se ter uma análise mais conclusiva de como essas variáveis podem influenciar na composição corporal e/ou, consequentemente, na avaliação da imagem corporal.

\section{Conclusão}

Este estudo mostrou que muitas adolescentes apresentaram insatisfação com sua imagem corporal, sendo evidente o desejo em obter uma silhueta menor que a atual. Todas as medidas de avaliação da composição corporal apresentaram associação significativa com a insatisfação corporal. Especificamente, o PC e a RCE foram as medidas mais condicionantes à manifestação de insatisfação corporal das adolescentes. O CS foi elevado tanto na avaliação do TT quanto do TC, sem associar-se com a insatisfação corporal.

A partir dos resultados encontrados podemos confirmar a necessidade da realização de estudos que avaliem diversos aspectos da imagem corporal em relação a diferentes medidas do estilo de vida que podem influenciar direta ou indiretamente o estado nutricional. Os comportamentos habituais adotados durante a adolescência podem ser mantidos durante a idade adulta, e juntamente com outros fatores de risco à saúde, aumentarem a incidência de distúrbios fisiológicos, psicológicos e sociais.

\section{Colaboradores}

NS Morais participou na concepção, na análise e interpretação dos dados, participou da elaboração do projeto, das coletas de dados e escrita do manuscrito e aprovou a versão final a ser publicada, VPN Miranda participou na concepção, da análise e interpretação dos dados, da elaboração do projeto, análise dos resultados e revisão da escrita do manuscrito e aprovou a versão final a ser publicada e SE Priore participou da concepção e desenho dos estudos, da coordenação do projeto e revisão do manuscrito como um todo e aprovou a versão final a ser publicada. 


\section{Agradecimentos}

À Fundação de Amparo à Pesquisa do Estado de Minas Gerais (FAPEMIG) e ao Conselho Nacional de Desenvolvimento Científico e Tecnológico (CNPq) pelo financiamento.

\section{Referências}

1. World Health Organization (WHO). Nutrition in adolescence: issues and challenges for the health sector: issues in adolescent health and development. Geneva: WHO; 2005.

2. Miranda VPN, Conti MA, Bastos RR, Laus MF, Almeida SS, Ferreira MEC. Imagem corporal de adolescentes de cidades rurais. Cien Saude Colet 2014;19(6):17911801.

3. Dohnt H, Tiggemann M. The Contribution of Peer and Media Influences to the Development of Body Satisfaction and Self-Esteem in Young Girls: A Prospective Study. Developmental Psychology 2006; 42(5):929-936.

4. Pelegrini A, Sacomori C, Santos MC, Sperandio FF, Cardoso FL. Body image perception in women: prevalence and association with anthropometric indicators. Rev Bras Cineantropom Desempenho Hum 2014; 16(1):58-65.

5. Cash TF. Cognitive-behavioral perspectives on body image. In: Cash TF, editor. Encyclopedia of body image and human appearance. Oxford: Elsevier; 2012. p. 334342.

6. Paludo AC, Pelegrini A, Grespan F, Caldeira AS, Madureira AS, Junior HS. Insatisfação com a imagem corporal em adolescentes: prevalência e associação com o estado nutricional. Conscientiae Saude 2011; 10(1):143-149.

7. Laus MF, Kakeshita IS, Costa TMB, Ferreira MEC, Fortes LS, Almeida SS. Body image in Brazil: recente advances in the state of knowledge and methodological issues. Rev Saude Publica 2014; 48(2):331-346.

8. Loomba-Albrecht LA, Styne DM. Effect of puberty on body composition. Curr Opin Endocrinol Diabetes Obes 2009; 16(1):10-15.

9. Miranda VPN, Faria FR, Faria ER, Priore SE. Maturação somática e composição corporal em adolescentes eutrófios do sexo feminino com ou sem adequação da gordura corporal. Revista Paulista de Pediatria 2014; 32(1):78-84.

10. Siervogel RM, Demerath EW, Schubert C, Remsberg KE, Siervogel, Chumlea WC, Sun S, Czerwinski AS, Towne B. Puberty and Body Composition. Hormones Research 2003; 60(Supl. 1):36-45.

11. Malina R, Bouchard C, Bar-Or O. Crescimento, maturação e atividade física. São Paulo: Phorte; 2009.

12. Moreno LA, Gottrand F, Huybrechts I, Ruiz JR, González-Gross, Dehenaw S ; HELENA Study Group. Nutrition and Lifestyle in European Adolescents: The HELENA (Healthy Lifestyle in Europe by Nutrition in Adolescence) Study. Adv. Nutr 2014; 5(5):615S-623S.

13. Saunders TJ, Chaput JP, Tremblay MS. Sedentary Behaviour as an Emerging Risk Factor for Cardiometabolic Diseases in Children and Youth. Canadian Journal of Diabetes 2014; 38:53-61.

14. Tenório MCM, Barros MVG, Tassitano RM, Bezerra J, Tenório JL. Atividade física e comportamento sedentário em adolescentes estudantes do ensino médio. Revista Brasileira de Epidemiologia 2010; 13(1):105-117.

15. Vasconcellos MB, Anjos LA, Vasconcellos MTL. Estado nutricional e tempo de tela de escolares da Rede Pública de Ensino Fundamental de Niterói, Rio de Janeiro, Brasil. Cad Saude Publica 2013; 29(4):713-722. 
16. Biddle SJH, Whitehead SH, O'Donovan TM, Nevil ME. Correlates of participation in physical activity for adolescent girls: a systematic review of recent literature. Journal Physical Activity and Health 2005; 2(4):423-434

17. Hallal PC, Knuth AG, Cruz DKA, Mendes MI, Malta DC. Pratica de atividade física em adolescentes brasileiros. Cien Saude Colet 2010; 15(2):3035-3042.

18. Farias Junior JC. Associação entre Prevalência de Inatividade Física e Indicadores de Condição Socioeconômica em Adolescentes. Revista Brasileira de Medicina do Esporte 2008; 14(2):109-114.

19. Ferrari EP, Gordia AP, Martins CR, Silva DA, Quadros TM, Petroski EL. Insatisfação com a imagem corporal e relação com o nível de atividade física e estado nutricional em universitários. Motricidade 2012; 8(3):52-58.

20. Laus MF, Almeida SS, Murarole MB, Braga-Costa TC Estudo de Validação e Fidedignidade de Escalas de Silhuetas Brasileiras em Adolescentes. Psicologia: Teoria e Pesquisa 2013; 29(4):403-409.

21. Kakeshita IS, Silva AIP, Zanatta IP, Almeida SS. Estudo de Validação e Fidedignidade de Escalas de Silhuetas Brasileiras em Adolescentes. Psicologia: Teoria e Pesquisa 2009; 25(2):263-270.

22. Conti MA, Cordás TA, Latorre MRDO. A study of the validity and reliability of the Brazilian version of the Body Shape Questionnaire (BSQ) among adolescents. Rev. Bras. Saude Matern. Infant. 2009; 9(3):331-338.

23. World Health Organization (WHO). WHO Expert Committee on Physical Status. Physical status: the use and interpretation of anthropometry. Geneva: WHO; 1995. WHO Technical Report Series n. 854.

24. Williams DP, Going SB, Lohman TG, Harsh DW, Srinivasan SR, Webber LS, Berenson GS. Body fatness and risk for elevated blood pressure, total cholesterol, and serum lipoprotein ratios in children and adolescents. Am J Public Health 1992; 82(3):358-363.

25. World health organization (WHO). Waist circumferences and waist-hip ratio: report of a WHO expert consultation. Geneva: WHO; 2008.

26. International Diabetes Federation - IDF [homepage on the Internet]. The IDF definition of the Metabolic Syndrome in children and adolescents 2007. [cited 2015 Dec 07]. Available from: http://www.idf.org/home.

27. Barros MVG, Nahas MV. Medidas da atividade física: teoria e prática em diversos grupos populacionais. Londrina: Midiograf; 2003.

28. Santos A, Andaki ACR, Amorim PRS, Mendes EL. Fatores associados ao comportamento sedentário em escolares de 9-12 anos de idade. Motriz 2013; 19(Supl. 3):S25-S34

29. Callegari-Jacques SM. Bioestatística: princípios e aplicações. Porto Alegre: Artmed; 2003.

30. Pagano M, Gauvreau K. Teoria da Amostragem. In: Pagano M, Gauvreau K. Princípios de bioestatística. $2^{\text {a }}$ ed. São Paulo: Cengage Learnig; 2008. cap. 22. p. 454-464.

31. Almeida SS, Nascimento PCBD, Quaioti TCB. Quantidade e qualidade de produtos alimentícios anunciados na televisão brasileira. Rev Saude Publica 2002; 36(3):353-355.

32. Pelegrini A, Petroski EL. Inatividade física e sua associação com estado nutricional, insatisfação com a imagem corporal e comportamentos sedentários em adolescentes de escolas públicas. Rev Paul Pediatr 2009; $27(4): 366-373$
33. Faria ER, Franceschini SCC, Peluzio MCG, Sant'Ana LFR, Priore SE. Correlação entre Variáveis de Composição Corporal e Metabólica em Adolescentes do Sexo Feminino. Arq Bras Cardiol 2009; 93(2):119-127.

34. Martins CR, Pelegrini A, Matheus SC, Petroski EL. Insatisfação com a imagem corporal e relação com estado nutricional, adiposidade corporal e sintomas de anorexia e bulimia em adolescentes. Rev Psiquiatr. Rio Grande do Sul 2010; 32(1):19-23.

35. Miranda VPN, Conti MA, Bastos R, Ferreira MEC. Insatisfação corporal em adolescentes brasileiros de municípios de pequeno porte de Minas Gerais. J Bras Psiquiatr 2011; 60(3):190-197.

36. Langoni POO, Aerts DRGC, Alves GG, Câmara SG. Prevalência de insatisfação com a imagem corporal e fatores associados em adolescentes escolares. Revista da Sociedade de Psicologia do Rio Grande do Sul 2012; 12(1):23-30

37. Pedroni JL, Rech RR, Halpern R, Marin S, Roth LR, Sirtoli M, Cavalli A. Prevalência de obesidade abdominal e excesso de gordura em escolares de uma cidade serrana no sul do Brasil. Cien Saude Colet 2013. 18(5):1417-1425.

38. Amaral ACS, Andrade MRM, Oliveira TP, Madeira RHA, Ferreira MEC. A cultura do corpo ideal: nível de satisfação corporal entre escolares de diferentes faixas etárias - estudo comparativo. HU rev. 2007; 33(2):4145.

39. Glaner MF, Pelegrini A, Cordoba CO, Pozzobon MA. Associação entre insatisfação com a imagem corporal e indicadores antropométricos em adolescentes. Rev Bras Educ Fís Esporte 2013; 27(1):129-136.

40. Corseuil MW, Pelegrini A, Beck C, Petroski ED. Prevalência de insatisfação com a imagem corporal e sua associação com a inadequação nutricional em adolescentes. Rev Educ Fís/UEM 2009; 20(1):25-31.

41. Petroski EL, Pelegrini A, Glaner MF. Motivos e prevalência de insatisfação com a imagem corporal em adolescentes. Cien Saude Colet 2012; 17(4):1071-1077.

42. Pereira EF, Graup S, Lopes AS, Borgatto AF, Daronco LSE. Percepção da imagem corporal de crianças e adolescentes com diferentes níveis socioeconômicos na cidade de Florianópolis, Santa Catarina, Brasil. Rev. Bras. Saúde Matern. Infant. 2009; 9(3):253-262.

43. De Onis M, Onyango AW, Borghi E, Siyam A, Nishida C, Siekmann J. Development of a WHO growth reference for school-aged children and adolescents. Bull World Health Organ 2007; 85(9):660-667.

44. Gonçalves VSS, Faria ER, Franceschini SCC, Priore SE. Predictive capacity of different bioelectrical impedance analysis devices, with and without protocol, in evaluation of adolescents. J. Pediatr. 2013; 89(6):567-574

45. Oliveira TC, Silva AAM, Santos CJN, Silva JS, Conceição SIO. Atividade física e sedentarismo em escolares da rede pública e privada de ensino em São Luís. Rev Saude Publica 2010; 44(6):996-1004.

Artigo apresentado em 10/12/2015

Aprovado em 01/09/2016

Versão final apresentada em 03/09/2016 
